\title{
Stress sensitivity of low permeability gas reservoir under different test conditions
}

\author{
Jian Yan ${ }^{1, ~ a ~, ~ A o j i a n g ~} \mathrm{Qi}^{2, \mathrm{~b}}$, Meitao $\mathrm{Fu}^{3, \mathrm{c}}$ \\ ${ }^{1,2,3}$ Shaanxi Key Laboratory of Advanced Stimulation Technology for Oil \& Gas Reservoirs, Xi'an \\ Shiyou University, Xi'an, 710065, China

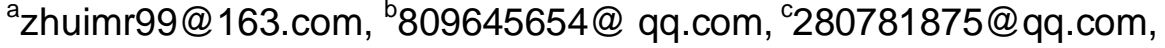

\begin{abstract}
Keywords: stress sensitivity; low permeability gas reservoir; triaxial stress; back pressure
Abstract: Past researches found that the results got by triaxial stress sensitivity experiments are near the fact of field, but the experimental conditions are not ascertain. This paper carried out triaxial stress sensitivity experiments under different testing conditions, such as back pressure, loading speed of confining pressure and the size of the axial stress. The results indicate that: relationship between net stress and dimensionless permeability follows exponential function; if there is back pressure on outlet of the testing cores, the stress sensitivity tested will be stronger; with the increase of axial stress, the stress sensitivity becomes weaker, to simulate the actual reservoir, the experiment should be carried out under the condition of triaxial stress; the faster the rising speed of the confining pressure, the more serious the stress sensitivity of the core, so proper loading speed should be selected when carrying out the stress sensitivity experiments. The studying results provide theoretical references for how carrying out stress sensitivity experiments.
\end{abstract}

\section{Introduction}

There are two main types of stress sensitivity evaluation methods, one kind is that changing the confining pressure to test the permeability with the confining pressure under the condition that the pore pressure is keeping constant; another kind is that keeping the confining pressure to the formation condition, then change the pore pressure and test the change of permeability. However, limited by the testing device, these two methods are carried out under the condition of single shaft. As it is known that the formation rock suffers three dimensional stresses: vertical stress and two horizontal stresses. The stress sensitivity under the condition of single shaft only considers the overlying formation pressure, so the testing results are not suited to the formation condition. So this paper carries out stress sensitivity experiments under triaxial stress, during the testing process, the testing conditions are changed, such as back pressure, loading speed of confining pressure and the size of the axial stress.

\section{Experimental equipment and method}

\section{Experimental equipment}
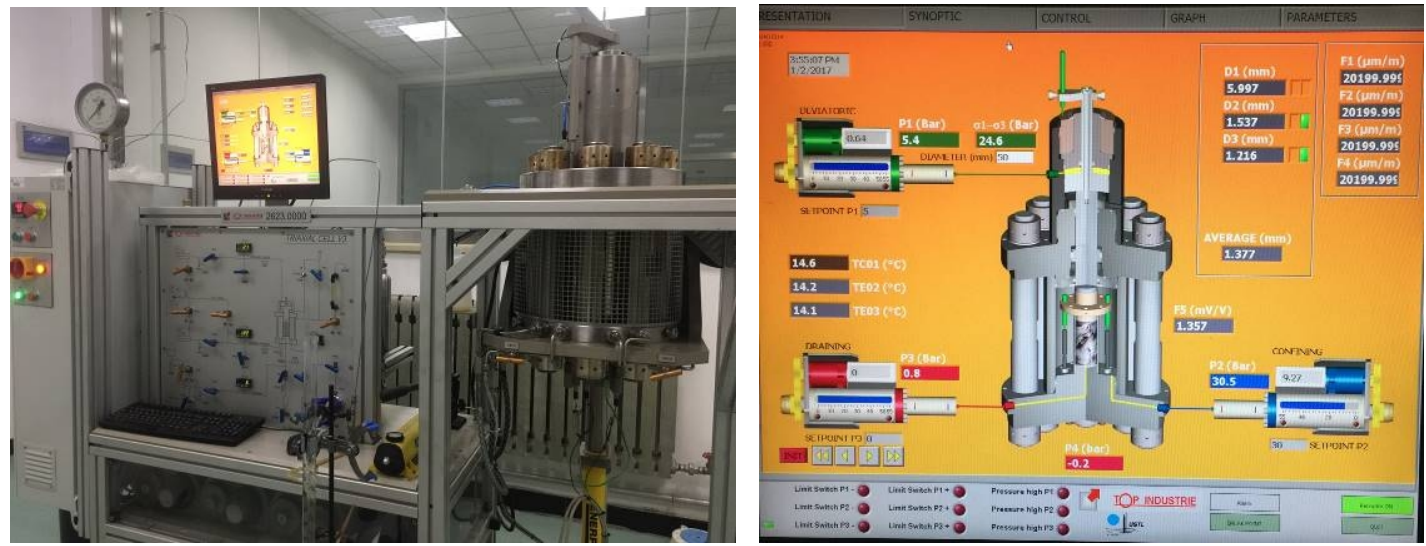

Fig.1 Triaxial stress sensitivity testing device(ROCK600-5HT)

Experimental equipment is the adaptive automatic triaxial testing machine (ROCK600-5HT), as shown in figure 1 , which is made by TOP INDUSTIE COMPANY. 
The testing system has the following characteristics: high pressure $\left(\mathrm{P}_{1 \max }: 100 \mathrm{MPa}, \mathrm{P}_{2 \max }: 50 \mathrm{MPa}\right.$, $\mathrm{P}_{3 \max }: 10 \mathrm{MPa}$ ); special strain ring, high precision; coupling the chemistry, mechanics and temperature; $\mathrm{Q}_{\max }$ of the pump: $15 \mathrm{~mL} / \mathrm{min}$; high testing efficiency and so on. The diameter of the testing samples can be $50 \mathrm{~mm}$ or $25 \mathrm{~mm}$. As the origin machine cannot test the flow rate of the gas, so a gas flow meter is added to test the flow rate.

Testing samples: the formation rocks are made in the shape of cylinder and the diameter of them are all $25 \mathrm{~mm}$. The permeability ranges from $2.35 \mathrm{mD}$ to $30.24 \mathrm{mD}$.

\section{Experimental methods}

There is no norm for testing the stress sensitivity by triaxial stress, so the testing method takes example by SY/T5358-2002.

Step1: Set back pressure $\mathrm{P} 4$ to be atmospheric pressure, axial pressure $\mathrm{P} 1$ to be $1 \mathrm{MPa}$, confining pressure $\mathrm{P} 2$ to be $3 \mathrm{MPa}$, and the pore pressure $\mathrm{P} 3$ to be $0.2 \mathrm{MPa}$, according to the tested flow rate of gas, calculate the permeability. Then change the confining pressure to be $6 \mathrm{MPa}, 9 \mathrm{MPa}, 12 \mathrm{MPa}$, $15 \mathrm{MPa}, 18 \mathrm{MPa}$, and test the corresponding permeability.

Step2: Set back pressure $\mathrm{P} 4$ to be $0.6 \mathrm{MPa}$, and change the $\mathrm{P} 3$ to be $0.7 \mathrm{MPa}$, then repeat the step1, compare the results with that of step 1 to analyze the effect of back pressure on testing permeability;

Step3: Set axial pressure $\mathrm{P} 1$ to be $2 \mathrm{MPa}, 3 \mathrm{MPa}, 4 \mathrm{MPa}$, repeats the step2, analyze the effect of axial pressure on the testing permeability;

Step4: Set the loading speed of confining pressure to be $0.15 \mathrm{MPa} / \mathrm{min}, 0.3 \mathrm{MPa} / \mathrm{min}$, $1.0 \mathrm{MPa} / \mathrm{min}$, and repeat the step 2 , analyze the effect of rising speed of confining pressure on testing permeability.

\section{Analysis of experimental results}

\section{Influence of back pressure}

As it is known that when the gas is flowing slowly in low permeability porous medium, an important phenomenon will appear, that is "gas slippage", Which will make the tested permeability larger than the absolute permeability. And with the decrease of the diameter of porous medium, the effect of gas slippage will be stronger. Two rock samples with different permeability are tested; the results are shown in figure 2 and figure 3 . It can be seen that whether the back pressure is low or high, the relationship between net stress and the dimensionless permeability (ratio between tested permeability and absolute permeability) follows exponential function. With the increase of the net pressure, the tested permeability decreases quickly, when the net pressure reaches to a certain value, the decreasing speed will become slow.

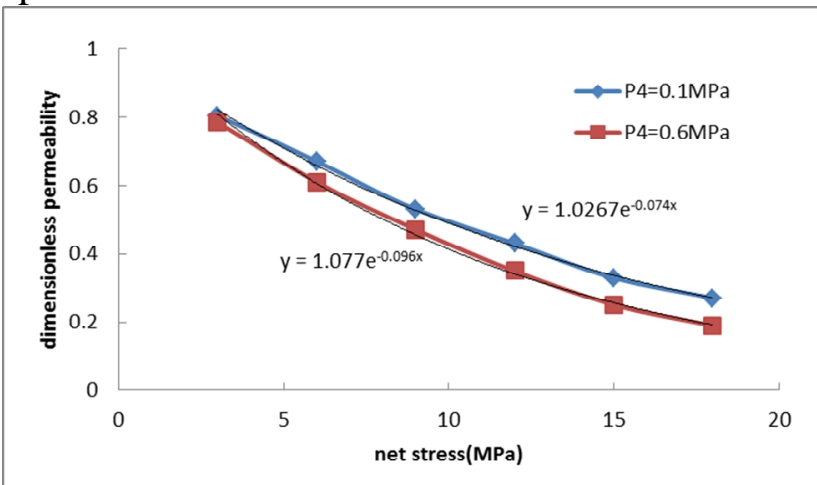

Fig.2 Relationship between net stress and dimensionless permeability $(K=29.16 \mathrm{mD})$

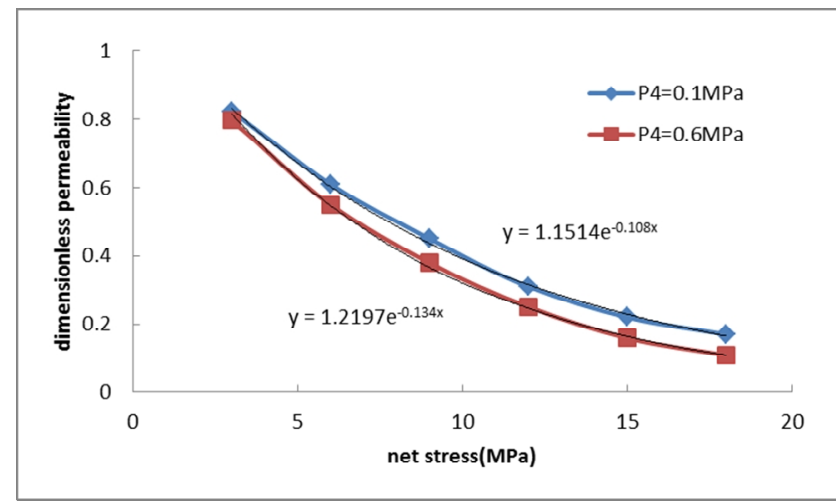

Fig. 3 Relationship between net stress and dimensionless permeability $(K=4.77 \mathrm{mD})$

The relationship between net stress and dimensionless permeability is as following: $\mathrm{k} / \mathrm{k}_{\mathrm{i}}=\beta \mathrm{e}^{-\alpha(\mathrm{pi}-\mathrm{p})}$ 
Where, $\mathrm{k}$ is the tested permeability; $\mathrm{k}_{\mathrm{i}}$ is the original permeability; $\beta$ is the revising coefficient, decided by lab experiments; $\alpha$ is the deformation coefficient; $p_{i}$ is original formation pressure; $p$ is the average current pressure.

From figure 2 and figure 3 , it also can be seen the difference between the stress sensitivity with back pressure and without. That is, if there is back pressure on outlet of the testing cores, the stress sensitivity tested will be stronger. The reason is that when there is back pressure, the pore pressure will be higher, which will weaken the effect of gas slippage, and make the tested permeability even smaller. And what's more, with the increase of net stress, the pore and pore throat of the core will become small, which will cause the gas slippage effect stronger, so it can be seen that: when the net stress increases, the difference becomes larger.

Compare the results of different permeability cores (Fig.4), another phenomena can be observed: the lower the permeability is, the more serious the stress sensitivity is.

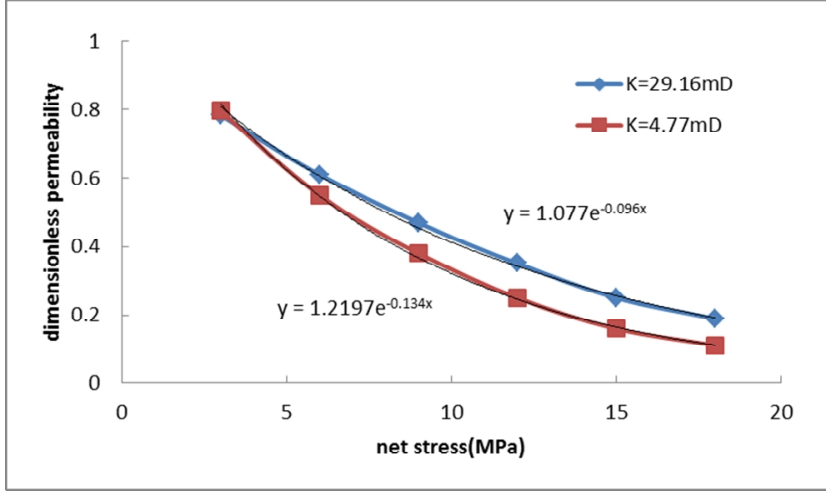

Fig. 4 Difference of stress sensitivity between different permeability rock

\section{Influence of axial stress}

The experimental results are shown in figure 5 and figure 6 , and the fitting relationships are listed in table 1 . They indicate that: with the increase of axial stress, the stress sensitivity becomes weaker, which can be seen obviously from the curves and the deforming coefficient $\alpha$. The reason is that, when the confining pressure increases, the net stress will rise, which will cause the radial deformation of the core and cause the length of core becoming longer, however, because of the axial stress, the axial displacement is restricted. So the deformation of the pore and pore throat becomes small when the axial stress increases, and the stress sensitivity appears weak. But with the increase of the axial stress, the stress sensitivity will be closer, for the deformation degree becoming small.
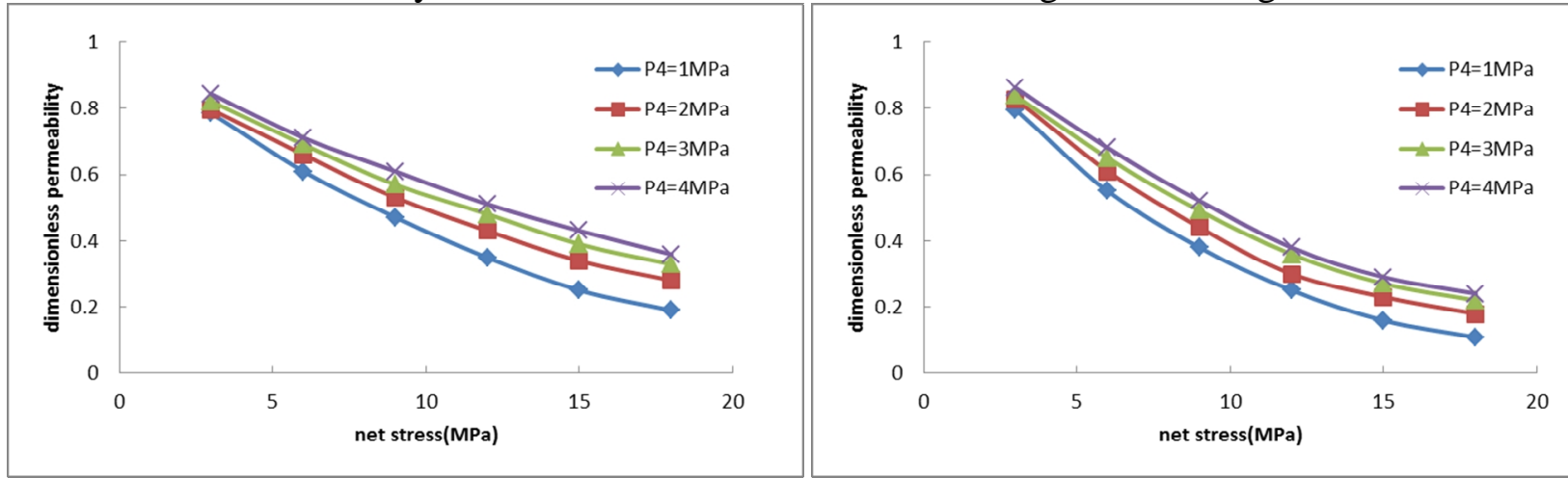

Fig.5 Relationship between net stress and dimensionless Fig. 6 Relationship between net stress and dimensionless permeability $(K=28.95 \sim 30.24 \mathrm{mD})$ permeability(K:4.56 4.77mD) 
Tab.l Fitting relationships dimensionless permeability and net stress

\begin{tabular}{|c|c|c|c|c|c|c|c|c|}
\hline \multirow{2}{*}{$\begin{array}{l}\text { Axial } \\
\text { stress } \\
(\mathrm{MPa})\end{array}$} & \multirow{2}{*}{$\begin{array}{l}\text { Core } \\
\text { number }\end{array}$} & \multirow{2}{*}{$\begin{array}{c}\text { Origin } \\
\text { permeability } \\
(\mathrm{mD})\end{array}$} & \multicolumn{2}{|c|}{ Fitting relationships } & \multirow{2}{*}{$\begin{array}{c}\text { Core } \\
\text { number }\end{array}$} & \multirow{2}{*}{$\begin{array}{c}\text { Origin } \\
\text { permeability } \\
(\mathrm{mD})\end{array}$} & \multicolumn{2}{|c|}{ Fitting relationships } \\
\hline & & & Formula & $\mathrm{R}^{2}$ & & & Formula & $\mathrm{R}^{2}$ \\
\hline 1.0 & 1 & 4.56 & $\mathrm{k} / \mathrm{k}_{\mathrm{r}}=1.078 \mathrm{e}^{-0.096(\mathrm{pi}-\mathrm{p})}$ & 0.997 & 5 & 29.16 & $\mathrm{k} / \mathrm{k}=1.220 \mathrm{e}^{-0.134(\mathrm{pi}-\mathrm{p})}$ & 0.998 \\
\hline 2.0 & 2 & 4.77 & $\mathrm{k} / \mathrm{k}_{\mathrm{r}}=0.997 \mathrm{e}^{-0.071(\mathrm{pi}-\mathrm{p})}$ & 0.996 & 6 & 30.15 & $\mathrm{k} / \mathrm{k}=1.070 \mathrm{e}^{-0.101(\mathrm{pi}-\mathrm{p})}$ & 0.995 \\
\hline 3.0 & 3 & 4.61 & $\mathrm{k} / \mathrm{k}_{\mathrm{s}}=0.992 \mathrm{e}^{-0.061(\mathrm{p}-\mathrm{p})}$ & 0.994 & 7 & 28.95 & $\mathrm{k} / \mathrm{k}=1.108 \mathrm{e}^{-0.092(\mathrm{pi}-\mathrm{p})}$ & 0.997 \\
\hline 4.0 & 4 & 4.83 & $\mathrm{k} / \mathrm{k}_{\mathrm{r}}=1.003 \mathrm{e}^{-0.057(\mathrm{p}-\mathrm{p})}$ & 0.995 & 8 & 30.24 & $\mathrm{k} / \mathrm{k}=1.131 \mathrm{e}^{-0.088(\mathrm{pi}-\mathrm{p})}$ & 0.996 \\
\hline
\end{tabular}

\section{Influence of loading speed of confining pressure}

The experimental results are shown in figure 7. The results indicate that: the faster the rising speed of the confining pressure, the more serious the stress sensitivity of the core. The reason can be explained by mechanics of materials: the faster the loading speed, the faster and more plastic deformation. While the more the plastic deformation, the smaller the pore and pore throat, which results the lower permeability. So when carrying out the stress sensitivity experiments, the proper loading speed should be selected, if loading so fast, the result of more serious stress sensitivity will be got.

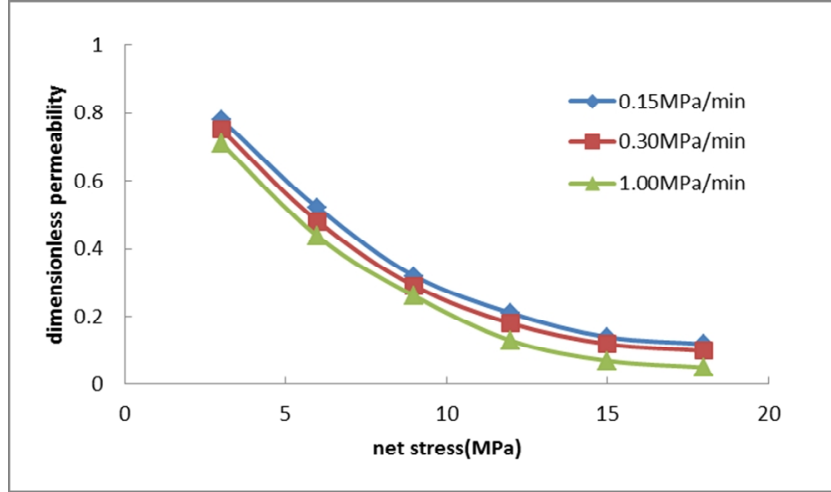

Fig.7 Effect of rising speed of confining pressure on stress sensitivity $(K: 2.35 \sim 2.47 \mathrm{mD})$

\section{Conclusion}

Through the experiment of triaxial stress sensitivity under different test conditions, it can be known that:

Relationship between net stress and dimensionless permeability follows exponential function;

If there is back pressure on outlet of the testing cores, the stress sensitivity tested will be stronger;

With the increase of axial stress, the stress sensitivity becomes weaker, to simulate the actual reservoir, the experiment should be carried out under the condition of triaxial stress;

The faster the rising speed of the confining pressure, the more serious the stress sensitivity of the core, so proper loading speed should be selected when carrying out the stress sensitivity experiments.

\section{Acknowledgments}

We would like to thank Shaanxi Key Laboratory of Advanced Stimulation Technology for Oil \& Gas Reservoirs in Xi' an Shiyou University for providing experimental conditions. In addition, it is supported by the national natural science fund item "Influence mechanism and correction of gas \& water relative permeability curve in tight gas reservoir" (51404197).

\section{References}

[1] Yan Jian etc. Stress sensitivity of low permeable and water-bearing gas reservoir without gas slippage effect [J]. Advanced Materials Research, 2014.06: 570-573 
[2] Liu Jiandong, Zhang Yurong etc. Research on the measurement of reservoir stress sensitivity under triaxial condition [J]. Complex Hydrocarbon Reservoirs, Volume 2, Issue 3, 2009:11-13

[3] ZENG Baoquan CHENG Linsong etc. Research on Stress Sensitivity Evaluation Experiments of Tight Sandstone Reservoir Based on Stress Sensitivity Constant [J]. Journal of Chongqing University of Science and Technology: Natural Science Edition. Volume 17, Issue 4, 2015:10-12

[4] ZHAO Feng, TANG Hongming etc. Damage characteristics and its evaluation technology of tight sandstone gas reservoirs [J]. Drilling \& Production Technology, Volume 34, Issue 5, 2011:47-51 